\title{
Composite period undulator to improve the wavelength tunability of free electron lasers
}

\author{
Takashi Tanaka* and Hideo Kitamura \\ RIKEN/SPring-8 Koto 1-1-1, Sayo, Hyogo 679-5148, Japan
}

(Received 2 February 2011; published 2 May 2011)

\begin{abstract}
A novel magnet configuration for permanent magnet undulators is proposed together with the operational procedures to improve the wavelength tunability of free electron lasers (FELs). The magnetization axes of the magnet blocks in the new configuration are inclined at a certain angle to those of the conventional Halbach configuration according to a particular rule. As a result, a composite-period magnetic field is generated, which contains two field components having two different periods, i.e., the fundamental and double periods. Moving the top and bottom magnet arrays toward the opposite directions along the undulator axis, it is possible to eliminate either of the two field components and thus the period is selectable. Simple analysis shows that the operation with the composite field is also useful for FELs despite that the gain length grows slightly due to the effects of the nonsinusoidal field profile. An example of the new undulator scheme applied to expanding the tunability of the SPring-8 x-ray FEL is presented together with discussions on technical issues.
\end{abstract}

DOI: 10.1103/PhysRevSTAB.14.050701

PACS numbers: 41.60.Cr, 41.60.Ap, 07.85.Qe

\section{INTRODUCTION}

The significant progress on accelerator technology in the past decade has opened a new possibility of free electron lasers (FELs) toward shorter wavelengths. Especially, the FELs operating in angstrom x-ray regions (XFELs) are considered to be the mainstream for the next generation synchrotron radiation (SR) sources. The Linac Coherent Light Source has achieved lasing at $0.15 \mathrm{~nm}$ [1], and Euro XFEL [2] and SPring-8 XFEL [3] are under construction aiming at lasing in harder $\mathrm{x}$ rays. Furthermore, there are a large number of FEL projects under proposal and construction, to be operated in short-wavelength regions from vacuum ultraviolet to angstrom $x$ rays [4].

In construction of the short-wavelength FEL facilities mentioned above, one of the most important issues is how to downsize the facility and thus reduce the total cost of construction. The most straightforward way is to reduce the electron energy required to achieve lasing at the desired wavelength, which results in reducing the accelerator length. This in turn imposes that the undulator period should be as short as possible and thus the in-vacuum undulators (IVUs) are necessarily chosen for this purpose [3,5].

One of the most important advantages of FELs against the conventional lasers is the wavelength tunability according to

$$
\lambda\left(\lambda_{u}, K\right)=\frac{\lambda_{u}\left(1+K^{2} / 2\right)}{2 \gamma^{2}},
$$

\footnotetext{
*ztanaka@spring8.or.jp

Published by the American Physical Society under the terms of the Creative Commons Attribution 3.0 License. Further distribution of this work must maintain attribution to the author(s) and the published article's title, journal citation, and DOI.
}

where $\lambda$ is the lasing wavelength, $\gamma$ is the Lorentz factor of the electron, $\lambda_{u}$ is the undulator period, and $K$ is the undulator deflection parameter also referred to as the $K$ value.

The wavelength tuning can be done by changing the electron energy and/or the $K$ value of the undulator. In facilities that accommodate more than one beam line and require the time sharing of the electron beam, changing the electron energy is not usually allowed to avoid interference between the beam lines in operation. In such a case, the tuning should be done only by adjusting the $K$ value.

Compared to the tunable region of undulator radiation in conventional SR facilities based on the spontaneous radiation, that of XFELs when operated at a fixed electron energy is quite limited. This is a consequence of the fact that the utilization of higher harmonic radiation is not promising, because the laser intensity is much weaker than the fundamental radiation as opposed to the spontaneous radiation. In addition, another point should be addressed in the case of compact XFEL facilities; a larger $K$ value is not expected due to the short undulator period, and thus the tunable range tends to be narrow when the electron energy is fixed. In this paper, we propose a novel magnet configuration for PM undulators and present how to operate it to extend the tunable range of FELs. In addition, comparisons with existing undulator schemes are made to reveal the advantages of the proposed scheme.

\section{PRINCIPLE}

Let us consider a magnet array as shown in Fig. 1(a), where an undulator magnet circuit over two periods is schematically illustrated. The magnetization vectors are inclined at 45 degrees to those of the conventional Halbach configuration [6]. It is worth noting that an 
(a)



(b)



(c)

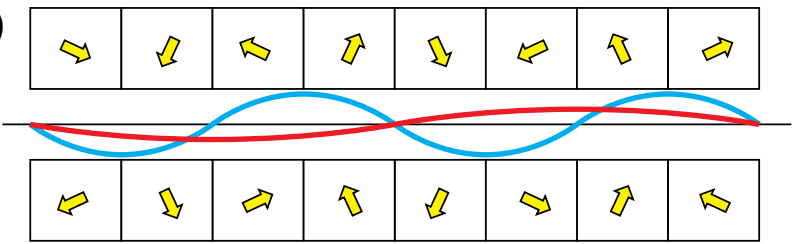

(d)



FIG. 1. Halbach-type undulator magnet circuits with four blocks per period (a) for the fundamental period and (b) for the double period. (c) Composite configuration of (a) and (b). (d) Two parameters to tune the photon energy: magnet gap $g$ and magnet shift $\Delta z$.

undulator with such a magnet configuration has been constructed and reported in [7]. Next let us consider a magnet array as shown in Fig. 1(b), which is obtained by rotating the magnetization vectors of Fig. 1(a) by $90^{\circ}$. Note that the rotation in the bottom array is done clockwise/counterclockwise in the left/right half and that in the top array is done in the opposite manner. It is easy to find that the magnetic field generated by this magnet array has a period of $2 \lambda_{u}$, the double of the original period. If the rotation angle $\theta$ is less than $90^{\circ}$, then we have a magnet array as shown in Fig. 1(c). The vertical magnetic field $B_{c}(z)$ generated by such a magnetic array is composed of two components having different periods of $\lambda_{u}$ and $2 \lambda_{u}$. To be more specific, it is given by

$$
B_{c}(z)=B_{1}(z) \cos \theta+B_{2}(z) \sin \theta \equiv B_{c 1}(z)+B_{c 2}(z),
$$

where $B_{1}(z)$ and $B_{2}(z)$ denote the magnetic distributions generated by the magnetic arrays in Figs. 1(a) and 1(b), respectively. It should be noted that the period of $B_{c}(z)$ is $2 \lambda_{u}$ and thus the fundamental energy of undulator radiation in this case is given by $\lambda\left(2 \lambda_{u}, K_{c}\right)$, where $K_{c}$ is the $K$ value corresponding to the magnetic field $B_{c}(z)$. It should be (a)

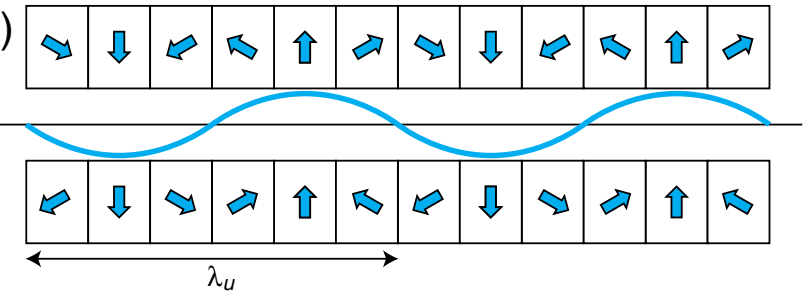

(b)



(c)

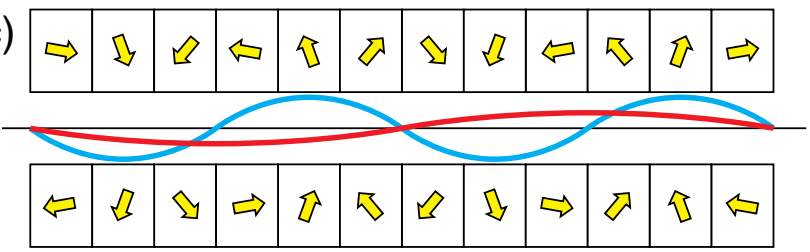

FIG. 2. As in Fig. 1 but with six blocks per period.

noted that $B_{c}(z)$ does not usually have a simple sinusoidal profile and thus $K_{c}$ should be calculated according to the mathematical form of the generalized $K$ value [8],

$$
K_{c}^{2}=2\left\langle[\xi(z)-\langle\xi(z)\rangle]^{2}\right\rangle,
$$

where $\xi$ is the normalized 1 st field integral defined as

$$
\xi(z)=\frac{e}{m c} \int B_{c}(z) d z
$$

where $e$ is the electron charge, $m$ is the electron rest mass, $c$ is the speed of light, and $\langle\cdots\rangle$ denotes the average over the length of $2 \lambda_{u}$.

In the above example, the number of blocks per period $(M)$ has been assumed to be 4 . As in the case of the conventional Halbach configuration, it is possible to increase $M$ to enhance the field amplitude. For example, Figs. 2(a) and 2(c) show the magnet configurations when $M$ of 6 has been chosen. For reference, an undulator with a magnet configuration shown in Fig. 2(a) has been constructed and reported in [9]. The undulator having the magnet array shown in Fig. 1(c) or 2(c) is hereinafter referred to as the composite-period undulator (CPU).

It should be noted that the above scheme cannot be applied directly to the hybrid undulator configuration, where pole pieces made of a magnetic material with high permeability are inserted between PM blocks to enhance the field amplitude. Instead of the hybrid configuration, the field enhancement by increasing $M$ is thus important when the requirement on the maximum $K$ value is stringent.

Now let us assume that the magnet arrays can be moved along the undulator axis, i.e., the longitudinal motion is available as well as the normal gap motion as shown in 
Fig. 1(d). This means that we have two parameters to play with to tune the wavelength, i.e., the magnet gap $g$ and magnet shift $\Delta z$. Taking into account the periodicity of $B_{c 1}$ and $B_{c 2}$, the magnetic field $B_{c}$ is approximately written in the form

$$
B_{c}(z, g, \Delta z)=B_{c 1}(z, g) \cos \left(\frac{\pi \Delta z}{\lambda_{u}}\right)+B_{c 2}(z, g) \cos \left(\frac{\pi \Delta z}{2 \lambda_{u}}\right) .
$$

The $K$ value is also written as follows:

$$
K_{c}^{2}(g, \Delta z)=K_{c 1}^{2}(g) \cos ^{2}\left(\frac{\pi \Delta z}{\lambda_{u}}\right)+K_{c 2}^{2}(g) \cos ^{2}\left(\frac{\pi \Delta z}{2 \lambda_{u}}\right)
$$

where $K_{c 1}$ and $K_{c 2}$ are the $K$ values corresponding to the field components $B_{c 1}$ and $B_{c 2}$, respectively.

Now let us explain how to operate the CPU. In principle, it is possible to select any combination of the two parameters $(g, \Delta z)$ as indicated by the yellow area in Fig. 3, where the two-dimensional (2D) space spanned by $g$ and $\Delta z$ is shown. Among them, we introduce three operation modes that are of practical importance, i.e., the fundamental, double-period, and composite modes, which are shown by line segments in Fig. 3.

In the fundamental and double-period modes, $g$ is varied from $g_{\min }$ to $g_{\max }$, whereas $\Delta z$ is kept constant at $\lambda_{u}$ and $\lambda_{u} / 2$, respectively. It is found from Eq. (3) that the undulator period of the fundamental mode is $\lambda_{u}$ and that of the double-period mode is $2 \lambda_{u}$. In addition, the maximum $K$ values in the fundamental and double-period modes are found to be $K_{c 1}\left(g_{\min }\right)$ and $K_{c 2}\left(g_{\min }\right) / \sqrt{2}$, respectively.

In the composite mode, $g$ is kept constant at $g_{\text {min }}$, while $\Delta z$ is varied from 0 to $\lambda_{u} / 2$. The undulator period of the composite mode is $2 \lambda_{u}$ and the $K$ value increases as $\Delta z$ decreases, and reaches a maximum of $\sqrt{K_{c 1}^{2}\left(g_{\text {min }}\right)+K_{c 2}^{2}\left(g_{\text {min }}\right)}$ when $\Delta z=0$.

Let us consider the field properties of the three operation modes in terms of the undulator field. Recalling that $B_{c 1}$

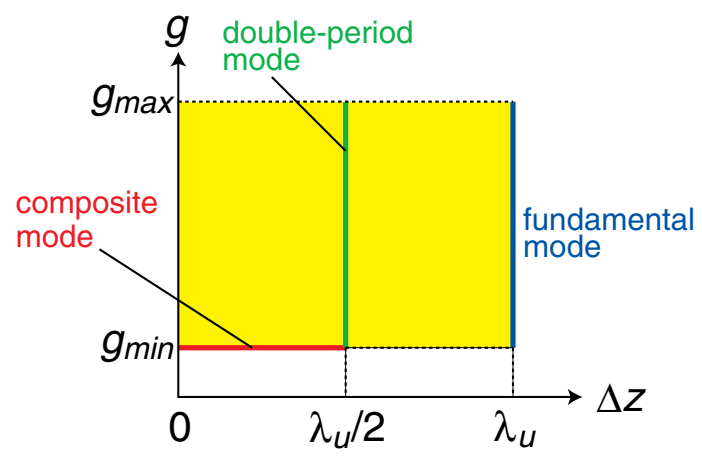

FIG. 3. Operation modes of the CPU. The magnet gap $g$ and shift $\Delta z$ are assumed to be varied from $g_{\min }$ and 0 to $g_{\max }$ and $\lambda_{u}$, respectively. The region painted yellow indicates the possible combination of $g$ and $\Delta z$, while the three line segments show the operation modes of practical importance. denotes the field component generated by the conventional Halbach configuration, it is found that CPU in the fundamental mode works as a simple undulator with a magnetic period of $\lambda_{u}$. In the same manner, we find that CPU in the double-period mode works as a simple undulator with a magnetic period of $2 \lambda_{u}$, except that the field distribution is not exactly sinusoidal. In contrast to these modes, the field distribution of the composite mode is far from a purely sinusoidal profile with a single period. It is thus important to explore the feasibility of the composite mode for the FEL application, which will be discussed in the next section.

\section{FEASIBILITY OF THE COMPOSITE MODE}

Let us consider the FEL process driven by the undulator having a periodic but nonsinusoidal field distribution. As an assumption, the undulator has only the vertical field component with a magnetic period of $\Lambda_{u}$. The 1D FEL equations are written as [10]

$$
\begin{aligned}
\frac{d \gamma_{j}}{d z} & =\frac{e}{m c^{2}} \beta_{x}\left(E e^{-i k_{u} z} e^{i \psi_{j}} e^{-i k_{u} z \Delta \omega / \omega_{0}}+\text { c.c. }\right), \\
\frac{d \psi_{j}}{d z} & =\frac{2 k_{u}\left(\gamma_{j}-\gamma_{0}\right)}{\gamma_{0}}-\frac{k_{u}}{1+K^{2} / 2}\left(\gamma_{j}^{2} \beta_{x}^{2}-\frac{K^{2}}{2}\right), \\
\frac{d E}{d z} & =-\frac{Z_{0}}{2} \beta_{x} j_{0} e^{i k_{u} z} e^{i k_{u} z \Delta \omega / \omega_{0}} N^{-1} \sum_{j=1}^{N} e^{-i \psi_{j}},
\end{aligned}
$$

where $\gamma_{j}$ and $\gamma_{0}$ are the Lorentz factors of the $j$ th electron and the average over the electron bunch, $\beta_{x}$ is the relative electron velocity in the horizontal direction, $E$ is the complex amplitude of the amplified radiation, $k_{u}=2 \pi / \Lambda_{u}$ is the undulator wave number, $\psi_{j}$ is the phase of the $j$ th electron, $K$ is the generalized deflection parameter of the undulator calculated by Eq. (2), $Z_{0}$ is the vacuum impedance, $j_{0}$ is the current density of the electron beam, $\Delta \omega$ is the detuning photon energy, and $N$ is the number of electrons in the range of interest.

Following the steps taken in [10], we have the differential equation on $E$ as follows:

$$
\frac{d^{3} E}{d z}-2 i k_{u} \frac{\Delta \omega}{\omega_{0}} \frac{d^{2} E}{d z}-\left(\frac{\Delta \omega}{\omega_{0}} k_{u}\right)^{2} \frac{d E}{d z}=i\left(2 \rho k_{u}\right)^{3} E,
$$

with

$$
\rho^{3}=\frac{e j_{0} Z_{0}|\kappa|^{2}}{8 m c^{2} \gamma_{0}^{3} k_{u}^{2}},
$$

where the parameter $\kappa$ denotes the interaction efficiency between the electrons and amplified radiation, which is defined as

$$
\begin{aligned}
\kappa & =\frac{1}{\Lambda_{u}} \int_{0}^{\Lambda_{u}} \xi(z) e^{i k_{u} z} e^{i \theta(z)} d z \\
\theta(z) & =\frac{k_{u}}{1+K^{2} / 2} \int\left[\xi^{2}(z)-K^{2} / 2\right] d z .
\end{aligned}
$$


Equation (5) has the conventional form of the FEL differential equation with the detuning photon energy of $\Delta \omega$ and it is easy to find that the radiation is amplified with the power gain length of $\Lambda_{u} /(4 \sqrt{3} \pi \rho)$ and the approximate bandwidth of $\rho$. Thus, the parameter $\rho$ is regarded to be the generalized Pierce parameter.

Assuming that the magnetic field of the undulator is purely sinusoidal with a period of $\Lambda_{u}$, we have

$$
\kappa=\frac{K}{2}\left[J_{0}\left(\frac{K^{2} / 4}{1+K^{2} / 2}\right)-J_{1}\left(\frac{K^{2} / 4}{1+K^{2} / 2}\right)\right] \equiv \kappa_{0},
$$

and $\rho$ reduces to the ordinary Pierce parameter.

Next let us calculate the FEL gain length when the vertical magnetic field is given in the form

$$
B_{c}(z)=B_{1 e} \cos \left(4 \pi z / \Lambda_{u}\right)+B_{2 e} \cos \left(2 \pi z / \Lambda_{u}\right),
$$

where $B_{1 e, 2 e}$ denote the effective amplitude of the field components with the periods of $\Lambda_{u} / 2$ and $\Lambda_{u}$, respectively. Then, the normalized field integral is given in the form

$$
\xi(z)=K_{1} \sin \left(4 \pi z / \Lambda_{u}\right)+K_{2} \sin \left(2 \pi z / \Lambda_{u}\right),
$$

with

$$
K_{1}=\frac{e B_{1 e} \Lambda_{u} / 2}{2 \pi m c}, \quad K_{2}=\frac{e B_{1 e} \Lambda_{u}}{2 \pi m c} .
$$

It is worth noting that Eq. (8) well approximates the horizontal relative velocity in the composite mode of CPU with $\lambda_{u}=\Lambda_{u} / 2$ and $K_{c}=\sqrt{K_{1}^{2}+K_{2}^{2}}$.

Under the above conditions, Eq. (7) is simplified to

$$
\kappa=\frac{1}{2 \pi} \int_{0}^{2 \pi} \xi(\varphi) e^{i \varphi} e^{i \theta(\varphi)} d \varphi,
$$

with

$$
\begin{aligned}
& \xi(\varphi)=K_{1} \sin 2 \varphi+K_{2} \sin \varphi \\
& \theta(\varphi)=\frac{1}{1+K_{1}^{2} / 2+K_{2}^{2} / 2} \int\left[\xi^{2}(\varphi)-K_{1}^{2} / 2-K_{2}^{2} / 2\right] d \varphi .
\end{aligned}
$$

This means that the interaction factor $\kappa$ is independent of $\Lambda_{u}$ and thus is given as a function of the two parameters $K_{1}$ and $K_{2}$, i.e., $\kappa\left(K_{1}, K_{2}\right)$. The gain length $L_{g}$ is then given by

$$
L_{g}\left(K_{1}, K_{2}\right)=L_{g 0}\left|\kappa\left(K_{1}, K_{2}\right) / \kappa_{0}\right|^{2 / 3},
$$

where $L_{g 0}$ is the gain length when $K_{1}=0$, i.e., the magnetic field is purely sinusoidal with the period of $\Lambda_{u}$.

By calculating the dependence of the gain length on the parameter $K_{1}$ with the deflection parameter $K_{c}$ being kept constant, we can investigate the effects due to the contribution of the field component with the period of $\Lambda_{u} / 2$. In Fig. 4, the gain length growth rate defined by $\left(L_{g}-L_{g 0}\right) / L_{g 0}$, which is obtained by numerical computation, is plotted as a function of $K_{1} / K_{2}$ for several different values of $K_{c}$. It is found that the gain length grows as $K_{1}$ increases, but the growth rate is not necessarily high as long as $K_{1} / K_{2} \leq 1$. For example, the growth rate is found to be

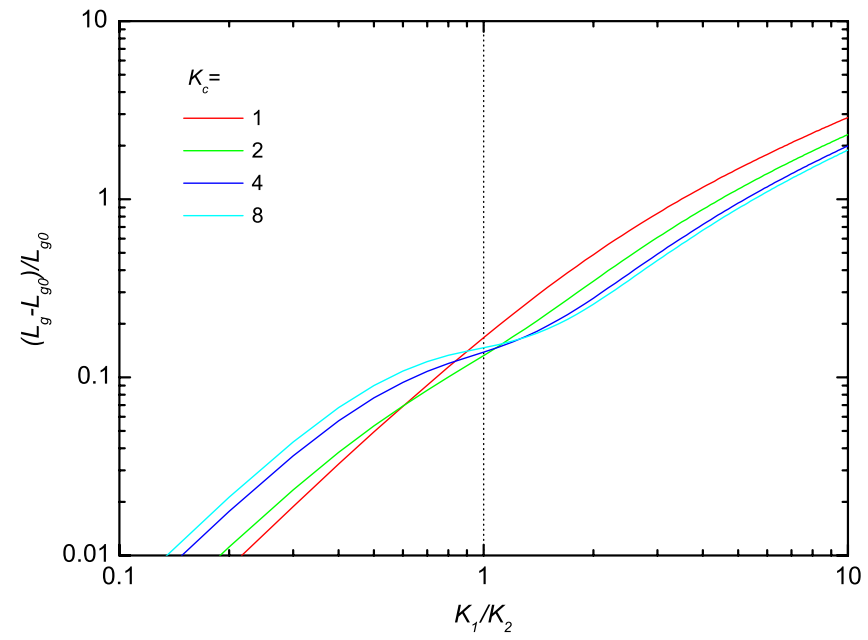

FIG. 4. Gain length growth due to the contribution of the half-period components for different values of $K_{c}$. Larger values of $K_{1} / K_{2}$ mean the larger contribution of the half-period components.

less than $10 \%$ even when $K_{1} / K_{2}=1 / 2$, i.e., $B_{1 e} / B_{2 e}=1$. In other words, the gain length grows by $10 \%$ when the purely sinusoidal field is contaminated by the half-period field component with the comparable amplitude. This is not a negligible number, but it still seems to be acceptable. Thus, we can conclude that the application of the composite mode to FELs is feasible enough.

\section{EXAMPLE}

In order to show how the CPU scheme extends the tuning wavelength of FELs, let us consider a CPU with the period of $18 \mathrm{~mm}$ and minimum gap of $3.5 \mathrm{~mm}$, which are the same as those of the IVUs for the SPring-8 XFEL. We assume that the PM material with the remanent field of 1.25 Tesla is available for IVUs. We also assume that the six-block configuration has been chosen to enhance the peak field. The rotation angle $\theta$ for the CPU configuration has been decided to be $20^{\circ}$ to optimize the FEL gain in the wavelength region as wide as possible.

Under the above assumptions, magnetic calculations have been carried out using the computer software RADIA [11], the results of which are shown in Fig. 5, for different values of the magnet shift at the minimum gap of $3.5 \mathrm{~mm}$. Note that the field distributions only within the central two periods are plotted for simplicity.

The magnetic field is found to be purely sinusoidal with the period of $18 \mathrm{~mm}$ in the fundamental mode $(\Delta z=18 \mathrm{~mm})$ as shown in the red line, while that in the double-period mode $(\Delta z=9 \mathrm{~mm})$ has the dominant period of $36 \mathrm{~mm}$ as shown in the blue line. In the composite mode (others), the field is composed of two components with the two periods of 18 and $36 \mathrm{~mm}$.

By the numerical computation based on Eq. (2), the maximum $K$ values for the three operation modes are 


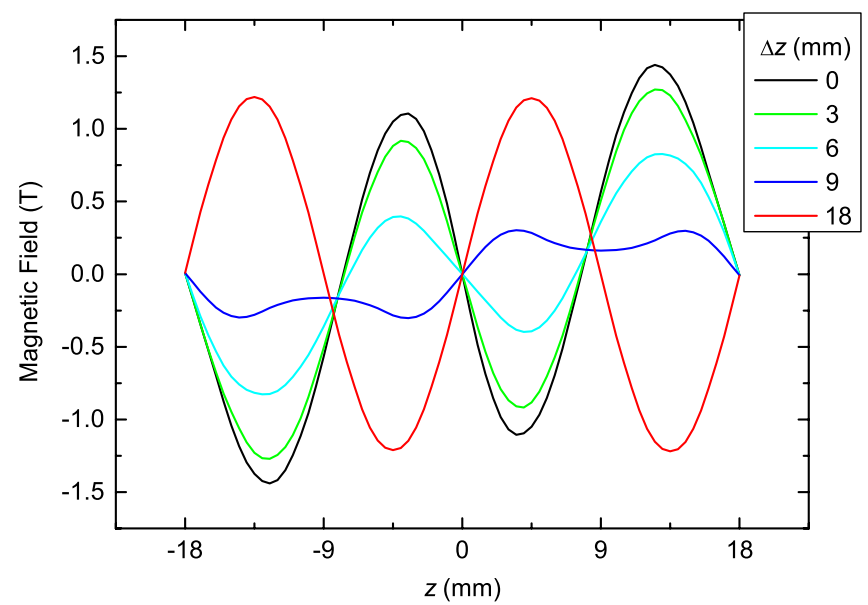

FIG. 5. Calculation results of the field distribution generated by the CPU. The blue and red lines show the field distributions in the double-period and fundamental modes, respectively.

found to be 2.05 (fundamental), 0.95 (double period), and 2.45 (composite). Recalling that the lasing wavelength is given by Eq. (1), we find that the longest wavelength in the double-period mode is shorter than that in the fundamental mode. This means that the double-period mode is useless in this example. It is worth noting that the maximum $K$ value in the case of the normal planar undulator, i.e., $\theta=0$, is found to be 2.18 , being $6 \%$ higher than that in the fundamental mode of the CPU.

Now let us explore the wavelength tunability of the FEL using the CPU described above. As an example, we take the electron-beam parameters of the SPring-8 XFEL as summarized in Table I. In the fundamental mode, it is easy to calculate the gain length using the universal function to denote the $3 \mathrm{D}$ effects derived in [12]. Namely,

$$
\frac{L_{g}}{L_{g, 3 \mathrm{D}}}=\frac{1}{1+\eta}
$$

where $L_{g}$ and $L_{g, 3 \mathrm{D}}$ are the $1 \mathrm{D}$ and 3D gain lengths, and the parameter $\eta$ denotes the gain degradation due to the 3D effects. According to [12], $\eta$ is given as a function of $L_{g}$ as well as the electron-beam and undulator parameters.

In the composite mode, we need to take into account the effects due to the nonsinusoidal field profile. To be more specific, we have to multiply $L_{g}$ by the growth

TABLE I. Electron-beam parameters for the gain length calculation.

\begin{tabular}{lc}
\hline \hline Electron energy & $8 \mathrm{GeV}$ \\
Peak current & $4 \mathrm{kA}$ \\
Normalized emittance & $0.7 \pi \mathrm{mm} \mathrm{mrad}$ \\
Energy spread & $5 \times 10^{-5}$ \\
Averaged betatron function & $30 \mathrm{~m}$ \\
\hline \hline
\end{tabular}



FIG. 6. Gain length as a function of the lasing wavelength in the fundamental (black line) and the composite (solid red line) modes. For reference, the gain lengths for the conventional undulators are also plotted in the solid green and dashed red lines. Refer to the text for details.

factor $|\kappa|^{2 / 3}$, which is obtained by the numerical computation as has been done in the previous section, and recalculate the $3 \mathrm{D}$ effects $\eta$. The results are shown in Fig. 6 as the tunability curve, i.e., the gain length as a function of the lasing wavelength.

The blue and red lines show the tunability curves in the fundamental and composite modes, respectively. Switching the operation mode at the wavelength of $0.11 \mathrm{~nm}$, wavelengths ranging from 0.055 to $0.29 \mathrm{~nm}$ can be covered with a single undulator at the fixed electron energy of $8 \mathrm{GeV}$. The longest gain length over this wavelength range is found to be around $6 \mathrm{~m}$. This means that the saturation length is about $110 \mathrm{~m}$ and we need the same or longer undulator length, which is still feasible.

It is of interest to compare the above tunability curves to those with the conventional Halbach undulator. The dashed red line indicates the tunability curve with the undulator having the period of $36 \mathrm{~mm}$. We find that the gain length grows due to the nonsinusoidal field effects in the composite mode but also find that the growth rate is negligible in the shorter wavelengths and is thus acceptable. The dashed blue line shows the tunability curve with the undulator having the period of $18 \mathrm{~mm}$ and maximum $K$ value of 2.18 , the maximum value for the conventional Halbach configuration as mentioned above. Note that most of the curve is hidden by the solid line except the wavelength longer than $0.11 \mathrm{~nm}$. We lose the tunability from 0.11 to $0.12 \mathrm{~nm}$ by modifying the undulator from the conventional to the CPU operated in the fundamental mode, but at the same time we gain the tunability from 0.11 to $0.29 \mathrm{~nm}$ by operating the CPU in the composite mode. Thus, we can conclude that the CPU operated in the two operation modes significantly improves the wavelength tunability of FELs. 


\section{DISCUSSION}

In this paper, we have proposed a novel undulator scheme to expand the wavelength tunability. It should be noted that there is another type of undulator for such a purpose; the revolver undulator (RU), in which more than one magnet array is installed on a rotary girder and the magnet array with a desired period can be selected by rotating the girder. An arbitrary combination of the magnet period is possible, however, the mechanical structure necessarily becomes much more complicated than the CPU.

The advantage of the CPU against the RU becomes more obvious when shortening the undulator period is considered to be the top priority. In such a case, the minimum gap should be as narrow as possible and thus the IVU will necessarily be chosen. The cryogenic permanent magnet undulator (CPMU) [13], in which the PM array is cooled down to a cryogenic temperature around $100 \mathrm{~K}$ to improve the magnet properties, would be another option in the near future toward the shorter period. It is easy to understand that applying the RU scheme to IVUs is quite challenging. It should be noted that an in-vacuum RU with a magnet length of $1 \mathrm{~m}$ has been successfully constructed in SPring8 [14]. Nevertheless, we have a lot of technical challenges to be overcome to extend the length to $4-5 \mathrm{~m}$, the reasonable length for XFEL applications. Moreover, applying the RU scheme to CPMUs seems to be much more impractical.

On the other hand, it is more feasible to apply the CPU scheme to IVUs. What we have to do is to establish a mechanism to move the magnet array not only vertically but also longitudinally to control the magnet gap and shift. The longitudinal motion itself is already realized in many undulator schemes as a phasing motion and thus we need to consider a system that is compatible to ultrahigh vacuum environment. The most straightforward way is to modify the conventional IVU so that the out-vacuum girder can be moved longitudinally. Then the in-vacuum girder holding the magnet array follows the longitudinal motion because it is connected to the out-vacuum girder through the flexible vacuum bellows. Note that the stroke of the bellows should be long enough to allow the possible vertical and longitudinal motions. It is also obvious that the above system can also be applied to CPMUs after a minor modification.

[1] P. Emma et al., Nat. Photon. 4, 641 (2010).

[2] M. Altarelli et al., XFEL: The European X-Ray Free-Electron Laser Technical Design Report, DESY 2006-097.

[3] T. Tanaka and T. Shintake, SCSS XFEL Conceptual Design Report (RIKEN Harima Institute, 2005).

[4] T. Shintake, in Proceedings of the 2007 Particle Accelerator Conference, Albuquerque, New Mexico, 2007 (IEEE, Albuquerque, NM, 1998), p. 89, and references therein.

[5] B. D. Patterson et al., New J. Phys. 12, 035012 (2010)

[6] K. Halbach, J. Phys. C 1, 211 (1983).

[7] T. Tanaka, K. Shirasawa, T. Seike, and H. Kitamura, in Proceedings of the 8th International Conference on Synchrotron Radiation Instrumentation, San Francisco, California, 2003, AIP Conf. Proc. No. 705 (AIP, New York, 2003), p. 227.

[8] T. Tanaka and H. Kitamura, J. Synchrotron Radiat. 8, 1221 (2001).

[9] G. Rakowsky, B. Bobbs, R. Burke, W. McMullin, and G. Swoyer, Nucl. Instrum. Methods Phys. Res., Sect. A 296, 597 (1990).

[10] L. H. Yu, S. Krinsky, R. L. Gluckstern, and J. B. J. van Zeijts, Phys. Rev. A 45, 1163 (1992).

[11] O. Chubar, P. Elleaume, and J. Chavanne, J. Synchrotron Radiat. 5, 481 (1998).

[12] M. Xie, in Proceedings of the Particle Accelerator Conference, Dallas, TX, 1995 (IEEE, New York, 1995), p. 183.

[13] T. Hara, T. Tanaka, H. Kitamura, T. Bizen, X. Marechal, T. Seike, T. Kohda, and Y. Matsuura, Phys. Rev. ST Accel. Beams 7, 050702 (2004).

[14] H. Kitamura, T. Bizen, T. Hara, X. Marechal, T. Seike, and T. Tanaka, Nucl. Instrum. Methods Phys. Res., Sect. A 467-468, 110 (2001). 